\title{
SUBSTANTIATION OF THE COMPOSITION OF A SEMI-SOLID DOSAGE FORM WITH A PROBIOTIC COMPONENT FOR USE IN DERMATOLOGY
}

\author{
Alina Soloviova $\bowtie$ \\ Department of Biotechnology ${ }^{1}$ \\ alina.soloviova@gmail.com \\ Halyna Kukhtenko \\ Department of Cosmetology and Aromology ${ }^{1}$ \\ Olha Kaliuzhnaia \\ Department of Biotechnology ${ }^{1}$ \\ ${ }^{I}$ National University of Pharmacy \\ 53 Pushkinskaya str., Kharkiv, Ukraine, 61002
}

$\triangle$ Corresponding author

\begin{abstract}
One of the important issues in the pharmaceutical development of a semisolid preparation for dermal use is the scientific and experimental justification for choosing the base-carrier of active substances.

The aim of this study was to experimentally substantiate the choice of rational combination of excipients in the development of a semisolid dosage form with a probiotic component for use in dermatology.

Materials and methods. Hydrophilic gelling agents were used as excipients in the study: Sepiplus 400, Aristoflex AVC, Carbopol 934, hydroxyethylcellulose HEC, sodium alginate. Physical and chemical, pharmaco-technological and microbiological methods were used to select the optimal basis. Colloidal stability was determined using a laboratory centrifuge at a speed of $6000 \mathrm{rpm}$ for $5 \mathrm{~min}$, an electronic thermometer and a water bath laboratory. Thermostability was determined in the thermostat at $(40 \pm 2){ }^{\circ} \mathrm{C}$ for 24 hours. Investigation of the rheological properties of the samples was performed using a Rheolab QC (Anton Paar, Austria) rheoviscometer using a system of coaxial cylinders C-CC27/SS. The kinetics of water absorption of the samples were studied by dialysis through a semipermeable Cuprophan membrane, Type $150 \mathrm{pm}$ at a temperature of $(34 \pm 2){ }^{\circ} \mathrm{C}$. The number of viable lactobacilli cells was determined by surface seeding on Petri dishes with dense MRS medium.

Research results. The results of the study of the number of viable lactobacilli cells showed that the lowest number of viable cells was in sample based on HEC. Tests for thermostability and colloidal stability showed that for sample No. 5 based on sodium alginate, stratification was observed in the experiment, which indicates its instability. These samples were excluded. Samples on Sepiplus 400 and Aristoflex AVC on a set of rheological features have advantages both consumer and technological; and sample on $t$ Aristoflex AVC has the best indicators of osmotic activity and the viability of cells in the dynamics for this study.

Conclusions. As a result of physical and chemical (organoleptic, colloidal and thermostability), pharmaco-technological (osmotic and structural and mechanical properties) and microbiological studies (number of viable lactobacilli cells) studies it was found that further work should be carried out with a sample based on gelling agent Aristoflex AVC, which has the most optimal performance in this development for a semisolid dosage form with a probiotic component for use in dermatology.
\end{abstract}

Keywords: skin microbiome, probiotics, dermatological diseases, semisolid dosage form, emulgel, gelling agents.

DOI: $10.21303 / 2504-5679.2021 .002181$

\section{Introduction}

The skin is a complex barrier organ consisting of a symbiotic relationship between microbial communities and host tissue through complex signals provided by innate and adaptive immune systems [1]. The skin is constantly exposed to various endogenous and exogenous factors physical, chemical, bacterial and fungal, which affect this balanced system, potentially leading to inflammatory skin conditions, including infections, allergies or autoimmune diseases [2]. The skin, as a link between the internal organs and the external environment, is always in contact with various substances and agents, including microorganisms that colonize the skin [3]. 
In skin care products, probiotics can help restore the skin's natural balance by ensuring that it is functioning properly and is replenished with the necessary nutrients to stay protected from harmful factors and fight the signs of aging and environmental damage. Probiotics are also an effective anti-inflammatory agent, helping to relieve redness, irritation, and skin diseases, including acne, rosacea and dry skin [4].

Therefore, the creation of effective means of correction and protection of human normoflora to maintain a healthy skin microbiome today is an urgent task.

Preliminary analysis of the literature and our own research to create a semisolid dosage form for dermal use, we have selected the following active ingredients: probiotic component Lactobacillus strains; regenerating, anti-inflammatory, reparative component - dexpanthenol; moisturizing and antimicrobial component - lactic acid.

Probiotic strains of Lactobacillus plantarum 8P-A3 and Lactobacillus fermentum 90T-C4 are classic production strains that form the basis of many domestic and foreign probiotics, mainly in the form of lyophilized biomass, which are used to treat dysbacteriosis of various etiologies in children and adults; are characterized by high antagonistic properties and specific activity and are safe for human use [5].

According to the literature, an adequate number of lactobacilli, which is responsible for the manifestation of positive mechanisms of action of these microorganisms, is $10^{7}-10^{9} \mathrm{CFU}$ (colonyforming units) $/ \mathrm{ml}$ (or CFU/g) [6]. It is this range that we have focused on when creating a semisolid preparation for skin application.

Deviations from normal skin acidity can be the cause or consequence of many serious disorders of barrier function [6]. Therefore, to enhance the antimicrobial properties of the developing dosage form and reduce the alkalinity of the environment, which is characteristic of dermatitis and acne (prevalence in the skin of an alkaline environment leads to the reproduction of bacteria capable of provoking acne [7]), lactic acid solution was administered in the preparation. It should be noted that lactic acid is a metabolite of the organism of the macroorganism and can be considered as a biologically safe product, which differs favourably from other antimicrobial substances [8]. In cosmetics lactic acid is used as an effective mild exfoliant and skin moisturizer, as it is part of its natural moisturizing factor [9]. We hypothesized that due to the physiological nature of lactic acid to probiotic cultures (lactic acid bacteria assimilate glycogen, breaking it down to endogenous lactic acid [10]) strains of lactic acid bacteria are sufficiently resistant to its action, which will allow the usage of lactic acid in conjunction with probiotic cultures in one dosage form.

We also proposed the introduction of dexpanthenol, which belongs to the group of «skin» vitamins, as a dermatoprotector due to its regenerating, anti-inflammatory, and reparative action. Also, the use of dexpanthenol together with a probiotic component in one dosage form is justified due to the physiological needs of lactobacilli: in addition to carbohydrates, lactobacilli need for their development various growth factors, including pantothenic acid and its derivatives [11, 12].

The amount of dexpanthenol and lactic acid solution $80 \%$ was selected based on the analysis of semisolid preparations for the treatment of dermatological diseases and our own previous studies on the combined use of selected components with lactobacilli and was $4 \%$ and $1 \%$, respectively $[13,14]$.

One of the important issues in the pharmaceutical development of a semisolid preparation for dermal use is the scientific and experimental justification for choosing the base-carrier of active substances, because only the right combination of active ingredients with the base can provide the necessary action, appropriate technological and consumer qualities of the drug [15].

The use as an active component of living cells of probiotic cultures determines the specificity of the created medicine and puts forward a number of requirements for the preparation of the dosage form. The basis and parameters of production of living biotherapeutic drugs, first of all, should ensure the viability of cells and the preservation of their probiotic activity [16]. Therefore, the main criterion for the selection of excipients in the semisolid dosage form being developed is to maintain the survival of lactobacilli and ensure the stability of the drug.

Another factor influencing the choice of auxiliary components for a drug with a probiotic component is the content in the dosage form of water, which leads to hydration of lyophilized 
biomass and cell proliferation in the composition, which, in turn, will contribute to the instability of the dosage form. When choosing the basis, it was taken into account that hydrophilic bases, in particular macrogols, have a hyperosmolar effect, which leads to dehydration of the cell and its osmotic shock [17]. In this case, these characteristics of hydrophilic bases limit their use.

That is why some researchers suggest the use of lipophilic ointment bases in the development of semisolid dosage forms with a probiotic component. But in the development of semisolid preparations should take into account the main pathogenetic factors whose interaction contributes to the manifestation of infectious and inflammatory diseases of the skin, including acne: sebaceous gland production, changes in keratinization, colonization of pathogenic microorganisms follicles and the release of inflammatory mediators [18]. That is, in such dermatological diseases, the increase in the amount of sebum, in which the skin surface becomes oily makes it inappropriate to use purely lipophilic ointment or cream bases. This can lead to overload of fatty components of already oily skin, $\mathrm{pH}$ shift to the alkaline side, clogging of pores and lack of positive effect of the active components of the drug. Also, when using lipophilic bases, another problematic question arises - how easily microorganisms can be released from the oil base applied to the skin and thus become metabolically active, sufficient to provide the necessary probiotic effects.

We consider it possible to overcome these shortcomings by developing a semisolid dosage form on an emulgel basis. Such base is a stable heterogeneous system and has the advantages of emulsion and gel [19]. The formation of gels is associated with the emergence and strengthening of bonds between macromolecules of polymers with the formation of a spatial network that holds almost the entire volume of the solvent, which is a significant factor in this development. The introduction of the probiotic component into the composition of the drug should be carried out through the oil phase, thereby stabilizing the lactobacilli. That is, when using hydrophilic solvents, the creation of an oil phase in which lactobacilli are incorporated will protect the cells from the action of moisture and a certain hyperosmolar action of the base's components.

Thus, the aim of our study was to experimentally substantiate the choice of rational combination of excipients in the development of a semisolid dosage form with a probiotic component for use in dermatology.

\section{Material and methods}

The research was performed from 2019 to 2021 at the Department of Biotechnology of the National University of Pharmacy. Hydrophilic gelling agents were used as excipients in the study: acrylic acid derivatives - Sepiplus 400 (polyacrylate-13-polyisobutene-polysorbate-20, «Seppic», France), Aristoflex AVC (copolymer of acrylamidopropylpropanesulfonic acid and vinylpyrrolidone, «Clariant Surfactants», Switzerland), Carbopol 934 (polymer of acrylic acid with allyl sucrose or allyl pentaerythritol, «Lubrizol», USA), cellulose derivative - hydroxyethylcellulose HEC («Ashland», Netherlands), polysaccharide - sodium alginate («BASF», Germany). The selected gelling agents have a wide working range of $\mathrm{pH}$ with the possibility of its adjustment, which is an advantage in this development due to the content in the composition of lactic acid, allow to obtain semisolid dosage forms without the use of high temperatures, which is important when administering a probiotic component, are compatible with many substances. The concentration of gelling agents in this series of studies was $2 \%$.

The results of our previous studies were used [19] in the preparation of emulgel samples. The introduction of the probiotic component in the form of lyophilized biomass of lactobacilli into the soft dosage form being developed, it is advisable to carry out through the oil phase - vegetable oil, by pre-dissolving it in polysorbate- 80 . This method causes high survival rates of lactobacilli, system stability and uniform particle distribution in the oil phase. Propylene glycol (10\%), in which dexpanthenol was dissolved, was added to all samples to give the semisolid dosage form high consumer properties and to prevent drying. The lactic acid solution was introduced into the hydrophilic phase.

Emulgel samples were prepared in common approaches according to the specificity of gelling agents, given that Sepiplus 400 and Aristoflex AVC are pre-neutralized substances and have emulsifying ability, HEC requires control of the rate of addition in order to ensure the completeness of the wettability of the particles, Carbopol 934 - neutralization (using a solution of 
triethanolamine) and together with HEC - the presence of an emulsifier, the function of which was performed by polysorbate- 80 .

Based on our goal, modern physical and chemical, pharmaco-technological and microbiological methods were used to select the optimal basis. Organoleptic parameters, thermal and colloidal stability, $\mathrm{pH}$ and structural and mechanical properties of the studied samples were determined. Colloidal stability was determined using a OPN-8 laboratory centrifuge at the rate of $6000 \mathrm{rpm}$ for $5 \mathrm{~min}$, an TP-101 electronic thermometer and a WB-4 water bath laboratory. Thermostability was determined by keeping the samples first in a TC $1 / 80 \mathrm{SPU}$ thermostat at $(40 \pm 2){ }^{\circ} \mathrm{C}$, then in a refrigerator at $(10 \pm 2)^{\circ} \mathrm{C}$ and at room temperature, in the absence of delamination.

Investigation of the rheological (structural and mechanical) properties of the samples was performed using a Rheolab QC rheoviscometer (Anton Paar, Austria) using a system of coaxial cylinders C-CC27/SS. The Rheolab QC rheometer is equipped with RheoPlus software, which allows to set the necessary experimental conditions (range of the gradient of the shear rate, the number of measuring points and the measurement time of one point). Rheological measurements were performed in three stages:

a) a linear increase in the gradient of the shear rate from $2 \mathrm{~s}^{-1}$ to $300 \mathrm{~s}^{-1}$ with 105 measuring points and the duration of the measurement point $1 \mathrm{~s}$;

b) a constant shift at a shear rate of $300 \mathrm{~s}^{-1}$, one measuring point lasting $1 \mathrm{~s}$;

c) a linear decrease in the gradient of the shear rate from $300 \mathrm{~s}^{-1}$ to $2 \mathrm{~s}^{-1}$ with 105 measurement points and the measurement duration of the point $1 \mathrm{~s}$.

The study was performed at $25 \pm 0.5^{\circ} \mathrm{C}$, the duration of thermostating of each sample was 30 minutes.

The flow point (boundary) was calculated using the software using a mathematical model of the Casson rheological flow. The coefficient of dynamic rarefaction (flow) was determined at shear rates of 2.0 and $10.6 \mathrm{~s}^{-1}$, corresponding to the shear rate of the palm when distributing the semisolid dosage form on the surface and the viscosity of the system at shear rates of 27.8 and $151 \mathrm{~s}^{-1}$ corresponding to the speeds during processing in the process of its manufacture. Based on the results obtained, the values of the coefficients of dynamic rarefaction of the system were calculated according to the formulas:

$$
K_{d 1}=\frac{\eta_{2.0}-\eta_{10.6}}{\eta_{2.0}} \times 100 \% ; K_{d 2}=\frac{\eta_{27.8}-\eta_{151}}{\eta_{27.8}} \times 100 \%
$$

$K_{d 1}, K_{d 2}$ - dynamic rarefaction coefficients; $\eta$ - structural viscosity at appropriate shear rates, Pa·s.

The calculation of mechanical stability (MS) was performed at a shear rate of $2.0 \mathrm{~s}^{-1}$, the value of MS is defined as the ratio of the shear stress to failure $\left(\tau_{1}\right)$ to the shear stress after failure $\left(\tau_{2}\right)$ :

$$
M S=\frac{\tau_{1}}{\tau_{2}} .
$$

The kinetics of water absorption by the studied samples were studied by dialysis through a semipermeable Cuprophan membrane, Type $150 \mathrm{pm}$ at a temperature of $(34 \pm 2){ }^{\circ} \mathrm{C}$, maintained by a thermostat. The XSP-128 ULAB biological microscope was used to visually examine the homogeneity of the samples.

The number of viable lactobacilli cells was determined by surface seeding on Petri dishes with dense MRS medium. 15 to $20 \mathrm{ml}$ of molten MRS agar with a temperature of about $45^{\circ} \mathrm{C}$ was added to each Petri dish. After solidification of the medium and drying in a laminar stream of sterile air, a precisely measured volume $(0.1 \mathrm{ml})$ of the prepared test sample was distributed over its surface. The cultures were incubated in a thermostat for 3 days at a constant temperature $(37 \pm 1){ }^{\circ} \mathrm{C}$. The arithmetic means of the number of colonies of lactobacilli grown on nutrient medium was calculated and the number of CFU in $1 \mathrm{ml}$ of sample was determined. To control and identify lactobacilli, the morphology of colonies and cells was observed under microscopy (biological microscope XSP-128 ULAB). The microorganisms corresponded to the taxonomic characteristics - the morphology of the colonies on the media and the morphology of the cells under microscopy were typical for lactobacilli. 


\section{Research results}

In order to select the optimal type of gelling agent, we prepared each sample according to the method recommended for a particular polymer, with the introduction of selected active substances and determined their physical and chemical, pharmaco-technological and microbiological parameters.

The critical parameter of the semisolid dosage form with the probiotic component is the viability of lactobacilli cells, so the first step in our study was to determine the number of viable cells in each sample (Table 1).

\section{Table 1}

The number of viable lactobacilli cells in the test samples

\begin{tabular}{ccc}
\hline Sample & Gelling agent & The number of viable cells*, CFU/ml \\
\hline No. 1 & Sepiplus 400 & $(3.05 \pm 0.02) \cdot 10^{7}$ \\
No. 2 & Carbopol 934 & $(4.54 \pm 0.01) \cdot 10^{7}$ \\
No. 3 & Aristoflex AVC & $(3.80 \pm 0.02) \cdot 10^{7}$ \\
No. 4 & HEC & $(2.11 \pm 0.03) \cdot 10^{6}$ \\
No. 5 & Sodium alginate & $(2.93 \pm 0.01) \cdot 10^{7}$
\end{tabular}

Note: * the permissible value of the quality indicator of the drug under development - not less than $10^{7} \mathrm{CFU} / \mathrm{ml}, n=5 ; \mathrm{P}=95 \%$; $(M \pm m)$ - confidence interval

The results of this series of experiments showed that the lowest number of viable cells was in sample No. 4 based on HEC and was $(1.11 \pm 0.03) \cdot 10^{6} \mathrm{CFU}$ in $1 \mathrm{ml}$ of sample, which is less than the allowable value of the quality indicator of the drug «number of viable cells». Therefore, this sample was excluded from further studies.

The next step was the analysis of organoleptic and physical and chemical parameters of the experimental samples (Table 2).

Table 2

Organoleptic and physical and chemical parameters of the samples

\begin{tabular}{|c|c|c|c|c|c|}
\hline No. & $\begin{array}{l}\text { Gelling agent/Organ- } \\
\text { oleptic and physico- } \\
\text { chemical parameters }\end{array}$ & $\begin{array}{c}\text { No. } 1 \\
\text { (Sepiplus 400) }\end{array}$ & $\begin{array}{c}\text { No. } 2 \\
\text { (Carbopol 934) }\end{array}$ & $\begin{array}{c}\text { No. } 3 \\
\text { (Aristoflex AVC) }\end{array}$ & $\begin{array}{c}\text { No. } 5 \\
\text { (Sodium alginate) }\end{array}$ \\
\hline 1 & $\begin{array}{l}\text { Description and homoge- } \\
\text { neity }\end{array}$ & $\begin{array}{c}\text { Homogeneous mass } \\
\text { of white color, with } \\
\text { a small number of air } \\
\text { bubbles, with a slight } \\
\text { specific odor, sticky, } \\
\text { low-viscosity }\end{array}$ & $\begin{array}{l}\text { Homogeneous mass } \\
\text { of white color, with } \\
\text { a large number of air } \\
\text { bubbles, with a slight } \\
\text { specific odor, sticky, } \\
\text { not viscous }\end{array}$ & $\begin{array}{c}\text { Homogeneous mass } \\
\text { of white color, with } \\
\text { a small number of air } \\
\text { bubbles, with a slight } \\
\text { specific odor, sticky, } \\
\text { non-viscous }\end{array}$ & $\begin{array}{l}\text { Homogeneous after } \\
\text { mixing mass of white } \\
\text { color, with a slight } \\
\text { specific odor, non- } \\
\text { sticky, low-viscosity }\end{array}$ \\
\hline 2 & Thermal stability & stable & stable & stable & unstable \\
\hline 3 & Colloidal stability & stable & stable & stable & unstable \\
\hline
\end{tabular}

All samples are homogeneous in consistency, have the same color and specific odor. Samples No. 2 and No. 3 with the addition of Carbopol 934 and Aristoflex AVC are sticky to the touch, which can help the gel to adhere to the skin. Sample No. 1 is inferior in density to others, sticky, capable of stretching, elongated without breaking. Tests for thermal stability and colloidal stability showed that for sample No. 5 based on sodium alginate, stratification was observed in the experiment, which indicates its instability. This led to the exclusion of this sample from further research.

Developing the composition of semisolid drugs, much attention is paid to the study of their rheological properties. This is due to the fact that with the help of these indicators the composition of excipients is substantiated to ensure primarily consumer indicators, the choice of parameters of the technological process of production is carried out, storage stability is investigated, etc. 
The study of rheological properties showed that samples No. 1, No. 2, No. 3 are heterogeneous emulsion systems, which are stabilized by the use of surfactants aimed at reducing the surface tension at the phase boundary, and by using structurants in the hydrophilic and lyophilic phase.

Rheograms of the flow of samples are shown in Fig. 1, 2, which shows that all samples show a plastic type of flow, because their flow begins after some shear stress. The numerical interval from the origin to the flow of the system quantifies the elastic properties of the samples, i.e., the systems behave as a viscoelastic solid. Under the action of increasing shear force, the systems begin to flow, during the flow of the studied samples, the viscosity decreases, which is characteristic of non-Newtonian fluids.

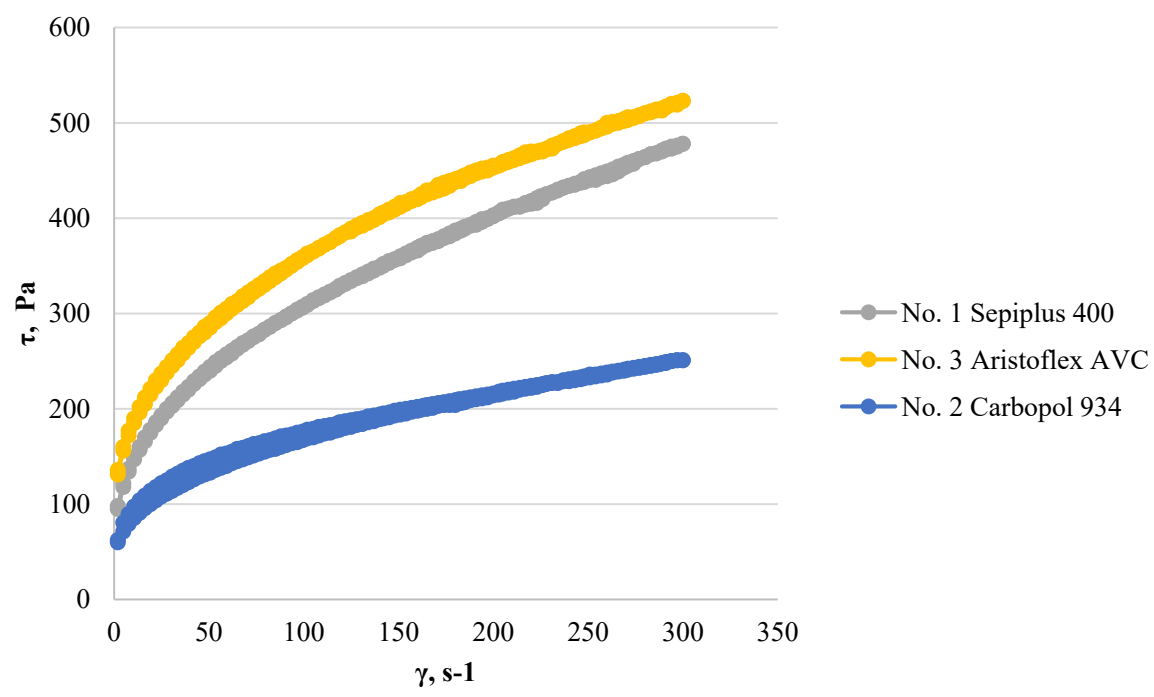

Fig. 1. Dependence of shear stress $(\tau, \mathrm{Pa})$ on the shear rate gradient $(\gamma, 1 / \mathrm{s})$ of the studied samples

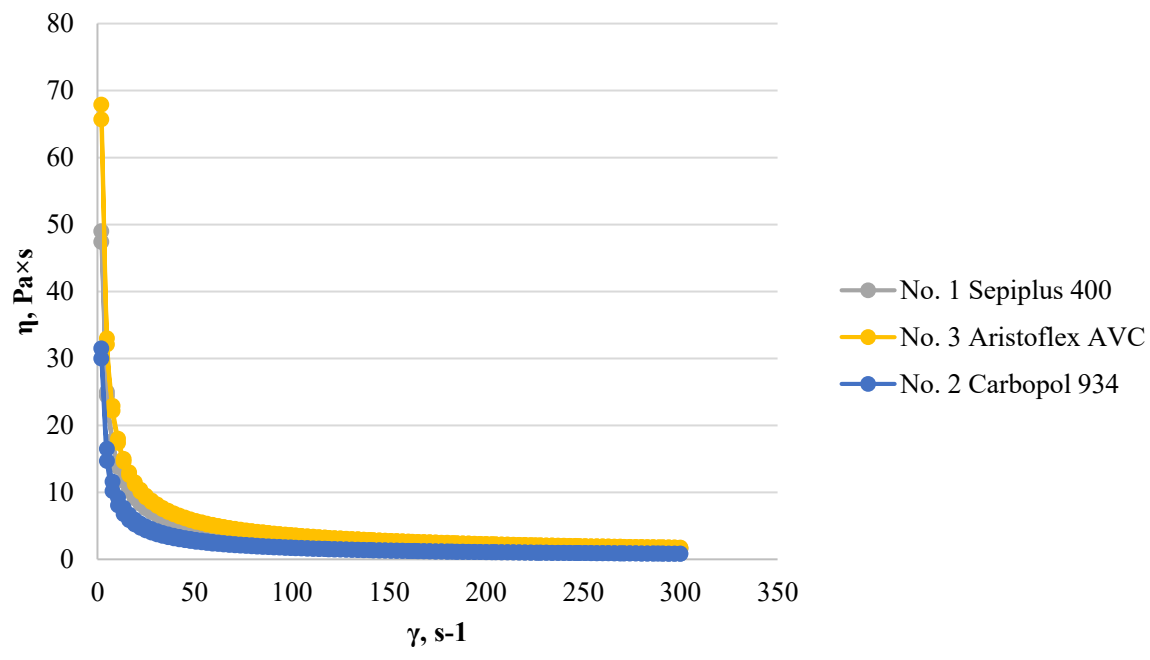

Fig. 2. Dependence of structural viscosity $(\eta, \mathrm{mPa} \cdot \mathrm{s})$ on the shear rate gradient of the studied samples

The profile of rheograms clearly shows the dependence of rheological parameters on the type of dispersed system, samples No. 1, No. 2, No. 3 with Sepiplus 400, Carbopol 934 and Aristoflex AVC, respectively, are highly thixotropic systems, the recovery of such systems coincides in time with their rarefaction, because the ascending and descending flow curves are practically superimposed on each other.

Tables 3, 4 show the values of shear stress and structural (dynamic) viscosity of the samples and the flow limits of the studied samples calculated by the mathematical model flow of the Casson I. 
Table 3

Values of shear stress and structural (dynamic) viscosity of samples

\begin{tabular}{|c|c|c|c|}
\hline $\begin{array}{l}\text { Shear speed gradient, } \\
\qquad\left(\gamma, \mathrm{s}^{-1}\right)\end{array}$ & $\begin{array}{c}\text { No. } 1 \\
\text { (Sepiplus 400) }\end{array}$ & $\begin{array}{c}\text { No. } 2 \\
\text { (Carbopol 934) }\end{array}$ & $\begin{array}{c}\text { No. } 3 \\
\text { (Aristoflex AVC) }\end{array}$ \\
\hline \multicolumn{4}{|c|}{ Shear stress $\left(\tau\right.$, Pa) with increasing/decreasing shear speed gradient $\left(\gamma, \mathbf{s}^{-1}\right)$} \\
\hline 2.0 & 98.1/94.8 & $62.3 / 59.9$ & $127 / 124$ \\
\hline 4.87 & $122 / 118$ & $80.5 / 71.3$ & $145 / 145$ \\
\hline 10.6 & $149 / 147$ & $97.5 / 85.4$ & $170 / 171$ \\
\hline 19.2 & $178 / 175$ & $114 / 100$ & 196/197 \\
\hline 27.8 & $200 / 197$ & $125 / 111$ & $216 / 217$ \\
\hline 151 & $358 / 360$ & $199 / 194$ & $356 / 359$ \\
\hline 300 & $478 / 478$ & $251 / 251$ & $454 / 453$ \\
\hline \multicolumn{4}{|c|}{ Structural (dynamic) viscosity $(\eta, P a \cdot s)$ with increasing/decreasing shear speed gradient $\left(\gamma, \mathrm{s}^{-1}\right)$} \\
\hline 2.0 & $49 / 47.4$ & $31.5 / 30$ & $63.2 / 61.9$ \\
\hline 4.87 & $25 / 24.4$ & $16.5 / 14.7$ & 29.9/4.87 \\
\hline 10.6 & $14.1 / 13.8$ & $9.2 / 8.06$ & $16.1 / 16.1$ \\
\hline 19.2 & $9.29 / 9.12$ & $5.93 / 5.21$ & $10.2 / 10.3$ \\
\hline 27.8 & $7.2 / 7.08$ & $4.52 / 4.01$ & $7.76 / 7.8$ \\
\hline 151 & $2.37 / 2.39$ & $1.32 / 1.28$ & $2.35 / 2.37$ \\
\hline 300 & $1.59 / 1.63$ & $0.83 / 0.83$ & $1.51 / 1.51$ \\
\hline
\end{tabular}

Table 4

Calculated structural and mechanical parameters of the samples

\begin{tabular}{lccc}
\hline \multicolumn{1}{c}{ Indicators } & $\begin{array}{c}\text { No. 1 } \\
\text { (Sepiplus 400) }\end{array}$ & $\begin{array}{c}\text { No. 2 } \\
\text { (Carbopol 934) }\end{array}$ & $\begin{array}{c}\text { No. 3 } \\
\text { (Aristoflex AVC) }\end{array}$ \\
\hline The flow limit by Casson I $\tau_{0}, \mathrm{~Pa}$ & 103.4 & 68.9 & 131.87 \\
Coefficient of dynamic rarefaction $K_{d 1}, \%$ & 71.22 & 70.79 & 73.52 \\
Coefficient of dynamic rarefaction $K_{d 2}, \%$ & 67.08 & 70.79 & 67.91 \\
Mechanical stability MC with $\gamma 2.0^{-1}$ & 1.04 & 1.04 & 0.96
\end{tabular}

Sample No. 2 with carbomer in the composition, has the lowest structural and mechanical properties, the system is easily liquefied under the action of low mechanical factors, which can be a critical parameter in scaling the production technology. Samples No. 1 Sepiplus 400 and No. 3 Aristoflex AVC on a set of rheological features have advantages both consumer and technological. Such systems are easy to apply and distribute on the skin surface, stable under the action of machining during production, used structurers Sepiplus 400 and Aristoflex AVC provide subjective cosmetic benefits to the developed samples.

Components used as components of bases are able to absorb water and have certain osmotic properties. It is known that the osmotic effect of hydrophilic bases can last 18 hours or more. This may prolong the action of the drug, but, as mentioned above, the same effect can lead to dehydration of skin cells and probiotics, which are the active ingredient of the drug. Therefore, the next step was to study the osmotic activity of the experimental samples (Fig. 3).

The study showed that during the experiment, the test samples absorb water gradually after 1 hour the mass of water absorbed for samples No. 1, No. 2 and No. 3 is $30 \%, 27.4 \%$ and $26.2 \%$, respectively, after 2 hours $-34.1 \%, 32.1 \%$ and $30.4 \%$, after 3 hours $-38.5 \%, 38 \%$, $35.1 \%$. Further study of the osmotic activity of the samples showed that the experimental samples No. 1 and No. 3 absorb water for 8 hours, and the sample No. 2 - for 10 hours. Then the samples reach equilibrium, with the lowest indicator of the level of absorption shows the sample No. 3 $62.1 \%$, samples No. 1 and No. 2 have indicators of $73.4 \%$ and $80 \%$, respectively.

Due to the need to preserve the survival of lactobacilli in the base, the next step was to determine the viability of cells in the experimental samples in the dynamics for 3 months (Fig. 4). 


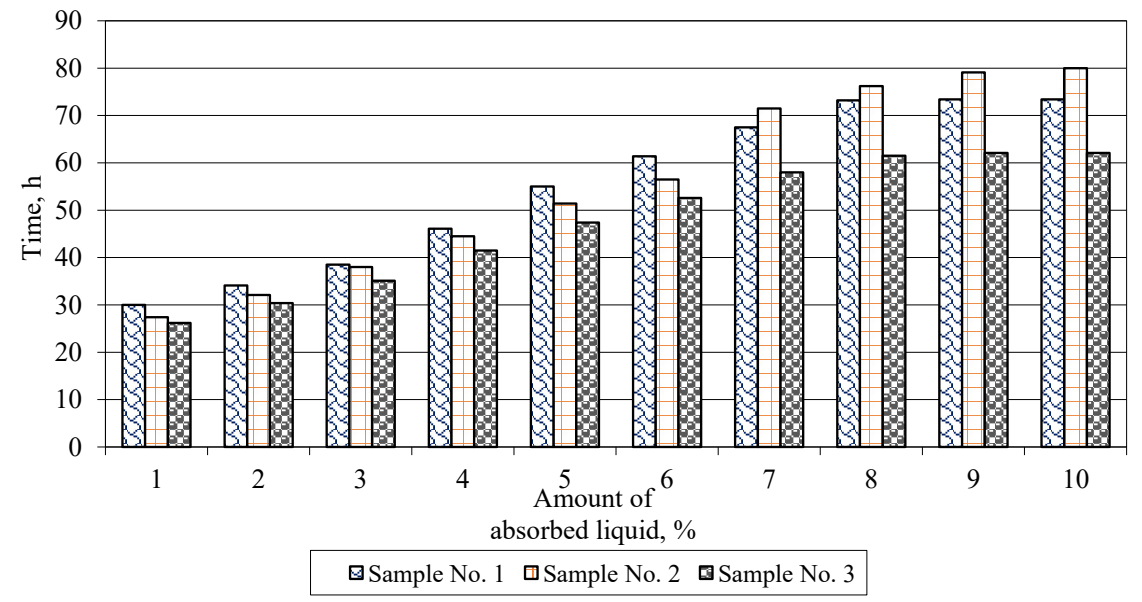

Fig. 3. Osmotic activity of experimental samples

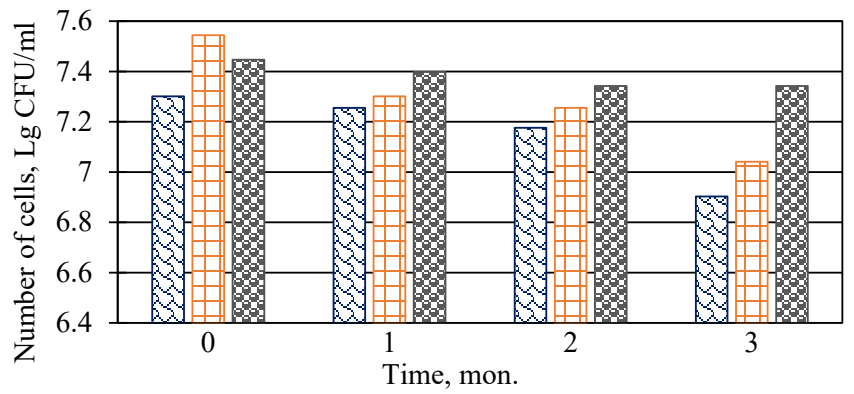

$\varpi$ Sample No. $1 \square$ Sample No. 2 固Sample No. 3

Fig. 4. Logarithm of the number of viable lactobacilli cells in the test samples

Previous studies have shown that in freshly prepared samples No. 1, No. 2 and No. 3 with the same initial dose of lactobacilli, the logarithm of the number of viable cells was 7.301, 7.544 and 7.447, respectively, i.e., the highest indicator was the sample based on Carbopol 934, the lowest - based on Sepiplus 400. But the determination of the number of viable cells in the dynamics for 3 months showed better characteristics for the sample based on Aristoflex AVC, the logarithm of the number of cells in $1 \mathrm{ml}$ of the sample was 7.342 and was higher than for the samples based on Carbopol 934 and Sepiplus 400 (7.041 and 6.903 respectively).

Thus, we focused on sample No. 3 based on Aristoflex AVC, which in this development has the best performance for a drug with a probiotic for use in dermatology.

\section{Discussion}

The skin microflora plays an important role in maintaining homeostasis and the state of local immunity. Current information on the state of the microbiome of healthy skin has led to the conclusion that detailing the composition of the microbiome in the sebaceous glands is normal and in pathology expands information about the pathogenesis of acne, seborrheic dermatitis and rosacea. Features of the microbiome of dry skin can be the basis for studying the pathogenesis of psoriasis, and areas with high humidity - to study the pathogenesis of atopic dermatitis [2]. Numerous studies show the need to maintain the skin microbiome [2,3]. Moreover, the violation of the skin microbiome can be a consequence of infectious and inflammatory dermatoses and their treatment with antimicrobials, and the cause, as the reduction of the microbiome of this ecosystem normally leads to the activation of opportunistic pathogens and, consequently, to infectious disease. And treatment of dermatoses exclusively with antimicrobial and anti-inflammatory drugs leads to long-term, often ineffective, treatment with frequent recurrences [20].

Therefore, for the treatment of this pathology, we have chosen a semisold dosage form for skin application, containing strains of probiotic cultures, dexpanthenol and lactic acid, to restore 
normal skin microbiocenosis and at the same time prevent or treat dermatological inflammatory and infectious diseases. That is, the product under development can be used as a primary treatment or, depending on the condition of the disease, as a concomitant during treatment and after to restore the skin, or as a prophylactic for skin types prone to these dermatological diseases.

In the studies, we prepared samples of a semisolid drug in the form of an emulgel based on Aristoflex AVC, Carbopol 934, Sepiplus 400, HEC and sodium alginate with active ingredients: lyophilized biomass of lactobacilli, dexpanthenol and lactic acid.

As a result of physical and chemical (organoleptic, colloidal and thermostability), pharmaco-technological (osmotic and structural and mechanical properties) and microbiological studies (number of viable lactobacilli cells) studies it was found that further work should be carried out with a sample based on gelling agent Aristoflex AVC, which has the most optimal performance in this development for a semisolid dosage form with a probiotic component for use in dermatology.

Study limitations. Concentration of gelling agent in these studies was $2 \%$, and it needs to be clarified among the range of possible concentrations.

Prospects for further research. The next step will be a pharmacological study of the adjusted emulgel composition and determine its stability during storage.

\section{Conclusions}

The criteria for selection of excipients in the creation of a semisolid dosage form with a probiotic component for dermatology were determined.

The expediency of creating a new drug with a probiotic in the form of an emulgel has been proved. The influence of gelling agents in the emulgel on the viability of lactobacilli cells, organoleptic and physicochemical parameters, on the rheological characteristics and on the absorption properties of the samples was studied.

Based on physical and chemical, pharmaco-technological and microbiological studies the rational composition of excipients in the composition of a new drug under the conditional name «Probioskin» was selected. According to the set of indicators, it is rational to use Aristoflex AVC as a gelling agent, which in this development is the most optimal for the drug for dermal application for the treatment and prevention of dermatological diseases.

\section{Conflict of interests}

The authors declare that they have no conflicts of interest.

\section{Financing}

The study was performed without financial support.

\section{Acknowledgments}

We would like to thank Leonid Strelnikov and Oksana Strilets for their encouragement and many fruitful discussions on this research.

\section{References}

[1] Byrd, A. L., Belkaid, Y., Segre, J. A. (2018). The human skin microbiome. Nature Reviews Microbiology, 16 (3), $143-155$. doi: http://doi.org/10.1038/nrmicro.2017.157

[2] Dréno, B., Araviiskaia, E., Berardesca, E., Gontijo, G., Sanchez Viera, M., Xiang, L. F. et. al. (2016). Microbiome in healthy skin, update for dermatologists. Journal of the European Academy of Dermatology and Venereology, 30 (12), $2038-2047$. doi: http://doi.org/10.1111/jdv.13965

[3] Tkachenko, N., Chagarovskyi, O., Dets, N., Sevastyanova, E., Lanzhenko, L. (2017). «Lving» and «probiotic» cosmetics: modern view and defenitions. Food Science and Technology, 11 (4). doi: http://doi.org/10.15673/fst.v11i4.735

[4] Fuchs-Tarlovsky, V., Marquez-Barba, M. F., Sriram, K. (2016). Probiotics in dermatologic practice. Nutrition, 32 (3), $289-295$. doi: http://doi.org/10.1016/j.nut.2015.09.001

[5] Drissi, F., Raoult, D., Merhej, V. (2017). Metabolic role of lactobacilli in weight modification in humans and animals. Microbial Pathogenesis, 106, 182-194. doi: http://doi.org/10.1016/j.micpath.2016.03.006 
[6] Niemeyer - van der Kolk, T., van der Wall, H. E. C., Balmforth, C., Van Doorn, M. B. A., Rissmann, R. (2018). A systematic literature review of the human skin microbiome as biomarker for dermatological drug development. British Journal of Clinical Pharmacology, 84 (10), 2178-2193. doi: http://doi.org/10.1111/bcp.13662

[7] Egert, M., Simmering, R., Riedel, C. (2017). The Association of the Skin Microbiota With Health, Immunity, and Disease. Clinical Pharmacology \& Therapeutics, 102 (1), 62-69. doi: http://doi.org/10.1002/cpt.698

[8] Tavaria, F. K. (2017). Topical use of probiotics: The natural balance. Porto Biomedical Journal, 2 (3), 69-70. doi: http://doi.org/ 10.1016/j.pbj.2017.02.003

[9] Wallen-Russell, C., Wallen-Russell, S. (2017). Meta Analysis of Skin Microbiome: New Link between Skin Microbiota Diversity and Skin Health with Proposal to Use This as a Future Mechanism to Determine Whether Cosmetic Products Damage the Skin. Cosmetics, 4 (2), 14. doi: http://doi.org/10.3390/cosmetics4020014

[10] Knackstedt, R., Knackstedt, T., Gatherwright, J. (2020). The role of topical probiotics in skin conditions: A systematic review of animal and human studies and implications for future therapies. Experimental Dermatology, 29 (1), 15-21. doi: http://doi.org/ 10.1111/exd.14032

[11] Ege, M. J. (2017). The Hygiene Hypothesis in the Age of the Microbiome. Annals of the American Thoracic Society, 14 (Supplement_5), S348-S353. doi: http://doi.org/10.1513/annalsats.201702-139aw

[12] Soloviova, A., Kaliuzhnaia, O., Strelnikov, L. (2020). Primary selection of the prebiotic components in the complex dermatological therapeutic and preventive medicine with probiotic. ScienceRise: Pharmaceutical Science, 2 (24), 33-39. doi: http://doi.org/ 10.15587/2519-4852.2020.201104

[13] Maguire, M., Maguire, G. (2017). The role of microbiota, and probiotics and prebiotics in skin health. Archives of Dermatological Research, 309 (6), 411-421. doi: http://doi.org/10.1007/s00403-017-1750-3

[14] Lee, G. R., Maarouf, M., Hendricks, A. J., Lee, D. E., Shi, V. Y. (2019). Topical probiotics: the unknowns behind their rising popularity. Dermatology Online Journal, 25 (5). doi: http://doi.org/10.5070/d3255044062

[15] Pelekh, I. R., Bilous, S. B. (2018). Modern approaches to use emulators and conservatives in the composition of dermatological drugs. Pharmaceutical Review, 3, 52-57. doi: http://doi.org/10.11603/2312-0967.2018.3.9453

[16] Derzhavna Farmakopeia Ukrainy. Vol. 2 (2021). Kharkiv: Derzhavne pidpryiemstvo «Ukrainskyi naukovyi farmatsevtychnyi tsentr yakosti likarskykh zasobiv», 424.

[17] McLoughlin, I. J., Wright, E. M., Tagg, J. R., Jain, R., Hale, J. D. F. (2021). Skin Microbiome - The Next Frontier for Probiotic Intervention. Probiotics and Antimicrobial Proteins. doi: http://doi.org/10.1007/s12602-021-09824-1

[18] Zuikina, S. S., Vyshnevska, L. I. (2020). The substantiation of the technology of the bigel hydrophilic faction for the complex therapy of mastopathy in the aspect of methodological approaches to drug creation. Social Pharmacy in Health Care, 6 (1), 31-39. doi: http://doi.org/10.24959/sphhcj.20.181

[19] Soloviova, A. V., Kaliuzhnaia, O. S. (2021). Selection of auxiliary components for the development of a soft preparation for probyotic components. Fundamentalni ta prykladni doslidzhennia u halizi farmatsevtychnoi tekhnolohii. Kharkiv: NFaU, 28-31.

[20] Akne. Klinichna nastanova, zasnovana na dokazakh (2017). Derzhavnyi ekspertnyi tsentr Ministerstva okhorony zdorovia Ukrainy, 101.

How to cite: Soloviova A., Kukhtenko H., Kaliuzhnaia, O. (2021). Substantiation of the composition of a semi-solid dosage form with a probiotic component for use in dermatology. EUREKA: Health Sciences, 6, 54-63. doi: http://doi.org/10.21303/ 2504-5679.2021.002181 